\title{
Automatic Recommendations for Data Coding: a use case from medical and teacher education
}

\author{
Claudia Schulz*, Michael Sailer ${ }^{\dagger}$, Jan Kiesewetter ${ }^{\ddagger}$, Elisabeth Bauer $^{\dagger}$, \\ Frank Fischer ${ }^{\dagger}$, Martin R. Fischer ${ }^{\ddagger}$, Iryna Gurevych* \\ ${ }^{*}$ Ubiquitous Knowledge Processing (UKP) Lab, Technische Universität Darmstadt, Germany \\ http://www.ukp.tu-darmstadt.de \\ ${ }^{\dagger}$ Chair of Education and Educational Psychology, LMU München, Germany \\ \{firstname.lastname\}@psy.lmu.de \\ ${ }^{\ddagger}$ Institute of Medical Education, University Hospital, LMU München, Germany \\ \{firstname.lastname\}@med.uni-muenchen.de
}

\begin{abstract}
Research in social sciences and humanities often involves analysing data to draw scientific conclusions. This however requires the manual coding of the data, which is highly time-consuming. A use case is the coding of students' essays in education to draw conclusions about students' reasoning and argumentation. The NeuralWeb API tackles this problem by providing automatic codings to other software components. These codings can for example be used in annotation platforms in terms of recommendations for expert coders from social sciences and humanities. After some initial manual annotations, the expert coders then merely need to verify the correctness of the automatic codings instead of manually annotating all data.
\end{abstract}

Index Terms-annotation recommendation, neural network API

\section{The NeURALWEB API}

To aid domain experts, such as social scientists and humanities researchers, with the time-consuming manual coding of textual data, we developed NeuralWeb. It provides an API that can be accessed by annotation platforms to receive automatic coding recommendations. In order to generate automatic codings, NeuralWeb requires a neural network model. Compared to non-neural machine learning methods, which require taskspecific feature engineering and linguistic knowledge, neural networks are easily applicable to any type of textual data. We chose a versatile Bi-LSTM architecture [1], which has proven useful for many tasks involving the identification of segments in texts, for example argument components [2]. It can however also be used for classification tasks, where each text is coded as belonging to one of various possible categories. Given a Bi-LSTM model trained on some manually coded texts, NeuralWeb receives un-coded texts via a POST request and replies with their automatic codings.

NeuralWeb was specifically developed to be used by annotation platforms that support the displaying of coding recommendations, such as INCEpTION ${ }^{1}$. Beyond this, NeuralWeb can be used by any software requiring the automatic coding of texts. This has the advantage of not having to integrate an automatic coding component into the software itself.

This work was supported by the German Federal Ministry of Education and Research (BMBF) under the reference 16DHL1040 (FAMULUS).

${ }^{1} \mathrm{https}: / /$ inception-project.github.io/

\section{Social SCIEnCES USE CASE}

The FAMULUS project ${ }^{2}$ constitutes the first use case of NeuralWeb, applying the API with regards to both coding recommendations and usage by other software. In this project, we consider data from teacher and medical education. Diagnostic reasoning is an important skill in many professions, which can be practised in simulations of diagnostic scenarios, e.g. the presentation of a (simulated) patient as part of medical education or a (simulated) pupil as part of teacher education. We collect diagnostic reasoning data in terms of students' textual explanations, in which they describe the reasoning underlying their diagnosis during these simulations.

To gain insights into students' diagnostic reasoning, the collected textual explanations are analysed with respect to various reasoning steps involved in diagnosis: generation of hypotheses, selection and evaluation of evidence, and drawing conclusions [3]. In other words, these steps need to be manually coded in the textual explanations. This is a highly time-consuming task, which can only be performed by domain experts in medicine and teacher education, who have the necessary domain knowledge to understand the explanations.

After manually coding the first half of the collected textual explanations, we tackled this problem by training a Bi-LSTM model on these codings and integrating it into NeuralWeb. For coding the second half of the textual explanations, the INCEpTION annotation platform [4] then accessed the NeuralWeb API to provide the domain experts with coding recommendations. Preliminary results indicate that this facilitated the manual coding, making it both easier and faster for the experts to code the texts. NeuralWeb furthermore provides the CASUS $^{3}$ e-learning and e-teaching software automatic codings of diagnostic reasoning, e.g. to show students their own reasoning structures within the e-learning environment. In current work, we extend the functionality of NeuralWeb to automatically train the Bi-LSTM model with manual codings received from INCEpTION. This allows domain experts without knowledge of Bi-LSTMs to use automatic codings for new coding tasks.

\footnotetext{
${ }^{2} \mathrm{http}: / /$ famulus-project.de

${ }^{3} \mathrm{https}: / /$ www.instruct.eu/
} 


\section{REFERENCES}

[1] N. Reimers and I. Gurevych, "Reporting Score Distributions Makes a Difference: Performance Study of LSTM-networks for Sequence Tagging," in Proceedings of the 2017 Conference on Empirical Methods in Natural Language Processing (EMNLP'17), 2017, pp. 338-348.

[2] C. Schulz, S. Eger, J. Daxenberger, T. Kahse, and I. Gurevych, "MultiTask Learning for Argumentation Mining in Low-Resource Settings," in Proceedings of the 2018 Conference of the North American Chapter of the Association for Computational Linguistics: Human Language Technologies (NAACL-HLT'18), 2018, pp. 35-41.

[3] F. Fischer, I. Kollar, S. Ufer, B. Sodian, H. Hussmann, R. Pekrun, B. Neuhaus, B. Dorner, S. Pankofer, M. R. Fischer, J.-W. Strijbos, M. Heene, and J. Eberle, "Scientific Reasoning and Argumentation: Advancing an Interdisciplinary Research Agenda in Education," Frontline Learning Research, vol. 4, pp. 28-45, 2014.

[4] J.-C. Klie, M. Bugert, B. Boullosa, R. E. de Castilho, and I. Gurevych, "The inception platform: Machine-assisted and knowledge-oriented interactive annotation," in Proceedings of the 27th International Conference on Computational Linguistics (COLING'18), 2018, pp. 5-9. 